\title{
YY1-C/EBP $\alpha$-miR34a regulatory circuitry is involved in renal cell carcinoma progression
}

\author{
WENHAO WENG ${ }^{1 *}$, MINLI WANG ${ }^{2 *}$, SUHONG XIE $^{3}$, YIN LONG $^{3}$, \\ FENG LI $^{3}$, FENYONG SUN ${ }^{1}$, YONGCHUN YU ${ }^{1}$ and $\mathrm{ZHI} \mathrm{LI}^{3}$

\begin{abstract}
${ }^{1}$ Department of Laboratory Medicine, Shanghai Tenth People's Hospital, Tongji University School of Medicine,
${ }^{3}$ Department of Laboratory Medicine, Shanghai Yangpu District Central Hospital, Shanghai 200090, P.R. China
\end{abstract} \\ Shanghai 200072; ${ }^{2}$ Department of Laboratory Medicine, Shanghai Putuo District Central Hospital, Shanghai 200013;
}

Received November 13, 2013; Accepted December 16, 2013

DOI: $10.3892 /$ or.2014.3005

\begin{abstract}
Renal cell carcinoma (RCC) is a common urological malignancy. It remains unclear, however, whether Yin Yang 1 (YY1) plays a functional role in the development of human RCC. In the present study, we demonstrated that levels of YY1 were significantly increased in primary RCC tissues when compared to these levels in the matched healthy tissues. YY1 knockdown inhibited cell growth, migration and invasion of RCC cells. Additionally, we highlighted a positive feedbackloop pathway resulting in YY1 upregulation. We observed that overexpression of YY1 caused repression of $\mathrm{C} / \mathrm{EBP} \alpha$ and the inhibition of $\mathrm{C} / \mathrm{EBP} \alpha$ led to the suppression of miR-34a. Since YY1 is a direct target of miR-34a, the low level of miR-34a increased the expression of YY1, promoting the aggressiveness of RCC cells. Furthermore, this feedforward mechanism was found in RCC tissues. We observed that miR-34a was downregulated in the pools of cancer tissues when compared to that of the normal tissues. The expression of miR-34a displayed an inverse correlation with YY1, but a positive correlation with $\mathrm{C} / \mathrm{EBP} \alpha$. In conclusion, our study highlights the importance of a novel regulatory circuitry for YY1 activation in maintaining the aggressive phenotypes of RCC.
\end{abstract}

\section{Introduction}

Renal cell carcinoma (RCC) is the most lethal of all urological malignancies, accounting for $\sim 2 \%$ of cancers worldwide (1). In the US, there are $\sim 65,000$ new cases of kidney cancer along with 13,500 deaths each year, with the majority of these

Correspondence to: Professor Zhi Li, Department of Laboratory Medicine, Shanghai Yangpu District Central Hospital, Shanghai 200090, P.R. China

E-mail: lizhiweng2010@163.com

*Contributed equally

Key words: YY1, miR-34a, C/EBP $\alpha$, renal cell carcinoma cases being related to RCC (2). Over the last four decades, the incidence of RCC has steadily increased (3). RCC shows less response to chemotherapy than other urological cancers (4). Surgical resection for clinically localized disease remains the mainstay for curative intervention. However, the aggressive and often insidious nature of RCC is reflected by recurrence rates of $20-40 \%$ after nephrectomy for clinically localized disease $(5,6)$. Thus, there is an urgent need to develop novel therapeutic approaches for RCC. To achieve this, a deeper understanding of the molecular and genetic networks that control the initiation and progression of RCC is imperative.

Yin Yang 1 (YY1) is a ubiquitous and multifunctional zinc-finger transcription factor member of the Polycomb group protein family, a group of homeobox gene receptors that play critical roles in normal biological processes such as embryogenesis, differentiation, replication and cellular proliferation (7). YY1 exerts its effects on genes involved in these processes via its ability to initiate, activate, or repress transcription depending upon the context in which it binds. There are several types of YY1-related diseases, such as viral infection and cancers. YY1 itself was found to be upregulated in many cancer types, including lymphoma, breast, prostate, colon, ovarian, cervical, and brain cancers and leukemia (8). Moreover, in many human cancer types, YY1 expression levels were found to be significantly elevated in the metastatic tumor compared to its primary counterpart, supporting the potential role of YY1 in cancer development. It was shown that YY1 may regulate by both oncogenes and tumor-suppressor genes through different mechanisms depending on the tissue context (9-12), suggesting the dual potential of YY1 as tumor suppressor or oncogene in malignant cells. However, the role of YY1 in the development of RCC is not fully understood.

microRNAs (miRNAs) are a class of single-stranded, highly conserved non-coding RNAs of 18-24 nucleotides that regulate gene expression by messenger RNA (mRNA) degradation or translational repression (13). To date, more than 17,000 miRNAs in over 153 species have been identified (miRBase Sequence Database - release 17; www.miRbase.org), of which $\sim 1,400$ are found in humans. Most miRs are evolutionarily conserved and are often found in clusters (14). Recent studies have highlighted the role of miR-34a as a tumor suppressor in a number of tumor types including prostate cancer, hepatocellular carcinoma, 
neuroblastoma and colon cancer (15-19). Functionally miR-34a was found to affect tumor cell proliferation, apoptosis, senescence, invasion, metastasis and drug resistance (19-23).

In the present study, we found that expression of YY1 was upregulated in RCC samples when compared to its primary counterpart. We also demonstrated that silencing of YY1 reduced cell growth and invasion of RCC cells, supporting its oncogenic role in the development of RCC. Furthermore, we confirmed that YY1 is a target of miR-34a. The elevated expression of YY1 represses CCAAT/enhancer-binding protein $\alpha(\mathrm{C} / \mathrm{EBP} \alpha)$, which leads to further inhibiton of miR-34a. This positive feedback loop promotes YY1 activity, which contributes to the development of RCC.

\section{Materials and methods}

Tissue samples. The paired tissue samples from primary tumor and adjacent non-tumor sites were obtained from 14 RCC patients during surgery prior to any therapeutic intervention at the Shanghai Tenth People's Hospital, Tongji University. All of the samples were subsequently verified by histology. Informed written consent was provided by all of the patients. The study protocol was approved by the Ethics Committee of Shanghai Tenth People's Hospital. To identify the differential expression of miR-34a, YY1 and $\mathrm{C} / \mathrm{EBP} \alpha$ in tumor specimens versus adjacent non-tumor renal tissue, we pooled these samples in two subsets. Pool 1 contained 14 primary tumor samples. Pool 2 was generated from the matched 14 normal renal tissue samples.

Cell lines and cell culture. RCC cell lines, 786-0 and ACHN, were cultured in Dulbecco's modified Eagle's medium (DMEM) containing 10\% fetal bovine serum (FBS), $100 \mathrm{U} / \mathrm{ml}$ penicillin and $100 \mu \mathrm{g} / \mathrm{ml}$ streptomycin. All cells were maintained in $5 \% \mathrm{CO}_{2}$ atmosphere at $37^{\circ} \mathrm{C}$.

Transfections. Cells were transfected with $50 \mathrm{nM}$ miR-34a mimics and inhibitors (GenePharma Co., Ltd., Shanghai China), YY1-siRNA (Sigma-Aldrich Co., LLC, St. Louis, MO, USA), YY1 and C/EBP $\alpha$ plasmids (Genechem Co., Ltd., Shanghai, China) using Lipofectamine 2000 (Invitrogen Corp., Carlsbad, CA, USA), respectively. RNA and proteins were harvested $48 \mathrm{~h}$ after transfection.

Quantitative RT-PCR. Total RNA was extracted from the tissues or cultured cells following the indicated treatment using TRIzol (Invitrogen Corp.) method. qRT-PCR or Stemloop PCR (24) was performed using the SYBR Green PCR kit (Takara Biotechnology Co., Ltd., Dalian, China), Real-time PCR data for mRNA (YY1, C/EBP $\alpha$ ) and miRNA (miR34a) are expressed relative to glyceraldehyde 3-phosphate dehydrogenase (GAPDH) or U6, respectively. The primers used were described as follows: YY1 forward, 5'-CCT GGC ATT GAC CTC TCA GAT CCA-3' and reverse, 5'-GGG CAA GCT ATT GTT CTT GGA GCA-3'; C/EBP $\alpha$ forward, 5'-AAC ATC GCG GTG CGC AAG AG-3' and reverse, 5'-TTC GCG GCT CAG CTG TTC CA-3'. The stem-loop miR-34a RT primer was 5'-CTC AAC TGG TGT CGT GGA GTC GGC AAT TCA GTT GAG ACA ACC AG-3'; miR-34a forward, 5'-ACA CTC CAG CTG GGT GGC AGT GTC TTA GCT GG-3' and reverse, 5'-TGG TGT CGT GGA GTC G-3'. All reactions were performed in triplicate. The relative expression levels of target genes or miRNA were calculated using the $2^{-\Delta \Delta C t}$ method (25).

Luciferase reporter assay. The wild-type YY1-3'UTR region, generated by PCR amplification, was cloned into the pcheck-2 luciferase reporter plasmid (Promega, Madison, WI, USA). Specific primers were designed for this system. The forward primer (5'-CCA CTC GAG GCA TCT TCC AGA AGT GTG AT-3') was Xhol-site-linked and the reverse primer Notlsite-linked (5'-CCA GCG GCC GCC ATT CTA CAA CTG AGC ACC AC-3'). Mutation of the miR-34a binding site was generated by a PCR-based site-directed mutagenesis method, using wild-type YY1-3'UTR reporter plasmid as the template. For the reporter assay, ACHN cells were plated onto 24-well plates and transfected with wild-type or mutant YY1-3'UTR reporter plasmid and miR-34a mimics or inhibitors using Lipofectamine 2000. After transfection for $48 \mathrm{~h}$, cells were harvested and assayed with the Dual-Luciferase Reporter Assay System (Promega) according to the manufacturer's instructions. The tests were repeated in triplicate. Firefly luciferase activity was normalized to Renilla luciferase activity.

Western blotting. Proteins were resolved on an SDS/PAGE gel (10\% gel) and subjected to immunoblot analysis using monoclonal antibodies against YY1, C/EBP $\alpha$ or GAPDH (Epitomics, Burlingame, CA, USA). All of the antibodies were used at working concentration (1:1000) in PBS-T with 5\% non-fat milk. The membrane was further probed with HRP (horseradish peroxidase)-conjugated rabbit anti-(mouse IgG) (1:2000 dilution; Santa Cruz Biotechnology), and the protein bands were visualized using enhanced chemiluminescence (Amersham Pharmacia).

Migration and invasion assay. To measure the cell migration activity, Transwell assays were performed using Corning 8.0- $\mu \mathrm{m}$ Transwell ${ }^{\circledR}$ cell culture inserts (Corning Inc., Corning, NY, USA). Membranes were coated with purified fibronectin (Sigma) at a concentration of $10 \mu \mathrm{g} / \mathrm{ml}$. For assessment of invasion, 1:10 diluted Matrigel-coated Transwell inserts (BD Biosciences, San Jose, CA, USA) were used. After transfection with either siRNA or expression vectors in opti-MEM medium for $5 \mathrm{~h}$, cells were collected and resuspended in serum-free DMEM medium containing $0.1 \%$ bovine serum albumin (BSA). Subsequently, cells $\left(4 \times 10^{5} / \mathrm{ml}\right)$ were seeded in Transwell chambers. After $24 \mathrm{~h}$ of incubation, the cells on the upper surface of the filter were completely wiped away with a cotton swab. The cells on the lower surfaces of the membrane were fixed with $100 \%$ methanol, and counted under a microscope.

Cell proliferation assay. Cells were seeded into 96-well plates at an initial density of $1 \times 10^{5}$ cells/well. MTT solution (200 $\mu \mathrm{l})(5 \mathrm{mg} / \mathrm{ml}$, Alfa Aesar) was added to each well and incubated for $5 \mathrm{~h}$ at $37^{\circ} \mathrm{C}$. The supernatant was then discarded, and $100 \mu \mathrm{l}$ of dimethyl sulfoxide (DMSO, Sigma) was added to each well to dissolve the precipitate. After $30 \mathrm{~min}$ at room temperature, the plates were scanned spectrophotometrically with a microplate reader (Beckman Coulter) set at $595 \mathrm{~nm}$ to measure the absorbance. 

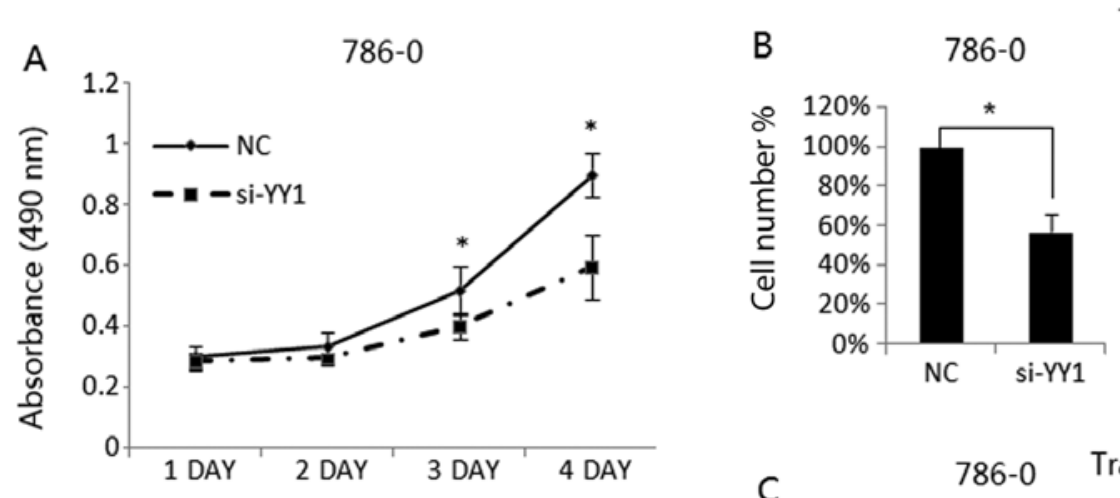

Transwell

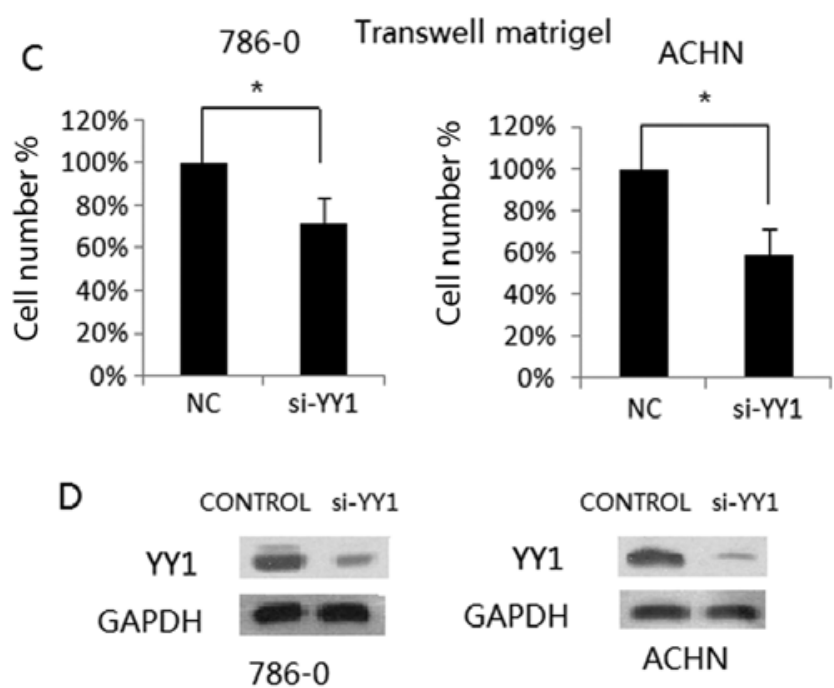

Figure 1. YY1 is critical for RCC cell growth, migration and invasion. (A) Knockdown of YY1 reduces cell proliferation as measured by MTT assays in control and ACHN or 786-0 cells following YY1 knockdown. P $<0.01$ analyzed by Student's t-test. Knockdown of YY1 reduces (B) cell migration and (C) invasion as measured by Transwell assays and Matrigel Transwell assays in control and ACHN or 786-0 cells following YY1 knockdown. P $<0.01$ analyzed by Student's t-test. (D) Validation of the knockdown efficiency of siRNAs. Western blots of YY1 protein in ACHN and 786-0 cells transfected with control or siRNAs targeting YY1 are indicated.

Statistical analysis. Statistical evaluations were conducted using the t-test. P-values $<0.05$ were considered to be statistically significant.

\section{Results}

Silencing of $Y Y 1$ inhibits RCC cell growth, migration and invasion. To confirm the tumor promotion effect of YY1 in RCC, we evaluated the impact of YY1 on the growth of RCC cell lines, 786-0 and ACHN. The growth curve of the RCC cell lines transfected with si-YY control siRNA is shown in Fig. 1A. The cell growth was significantly decreased in the si-YY1-transfected 786-0 and ACHN cells as compared with the control siRNA-transfected cells $(\mathrm{P}<0.05)$. To evaluated the impact of YY1 on cell migration and invasion, Transwell and Matrigel invasion assays were employed. We found that YY1 knockdown inhibited 786-0 and ACHN cell migration (Fig. 1B). Consistent with this finding, the Matrigel invasion assay showed that YY1 knockdown significantly inhibited the invasive capacity of RCC cells (Fig. 1C). These observations suggest that YY1 plays an important role in promoting cell growth, migration and invasive potential of RCC cells.

$Y Y 1$ is a direct target of miR-34a. To associate miRNAs with the regulation of YY1 expression, a bioinformatics search was performed for potential miRNAs targeting the mRNA of YY1 by using the public database Targetscan (www.targetscan. org). Noteworthy, there were several predicated miRNAs to target YY1, including miR-34a. miR-34a was reported to play a tumor-suppressive role in a number of tumor types (15-19). We then performed a luciferase reporter assay to verify that miR-34a directly targets YY1. We found that co-transfection of miR-34a mimics and wild-type YY1-3'UTR significantly decreased the luciferase activity in ACHN cells as compared with the control. However, miR-34a mimics had no effect on the luciferase activity when co-transfected with mutant YY1-3'UTR (Fig. 2B). These data demonstrate a specific inhibitory effect of miR-34a on the 3'UTR of YY1 through direct interaction. Furthermore, to examine the potential negative regulatory effect of miR-34a on endogenous YY1, we transfected miR-34a mimics, miR-34a inhibitors or control mimics into ACHN cells. In comparison with the miR control, we found the miR-34a exerted a discernible inhibitory effect on the YY1 protein level, while an increased protein level of YY1 was found in ACHN cells following transfection with miR-34a inhibitors (Fig. 2C). These data showed that YY1 is one of the direct targets of miR-34a.

Ectopic expression of miR-34a decreases the YY1-induced invasive or proliferative capacity of RCC cells in vitro. We next determined whether or not miR-34a overexpression impairs the oncogenic effect of YY1 on cell growth, migration 

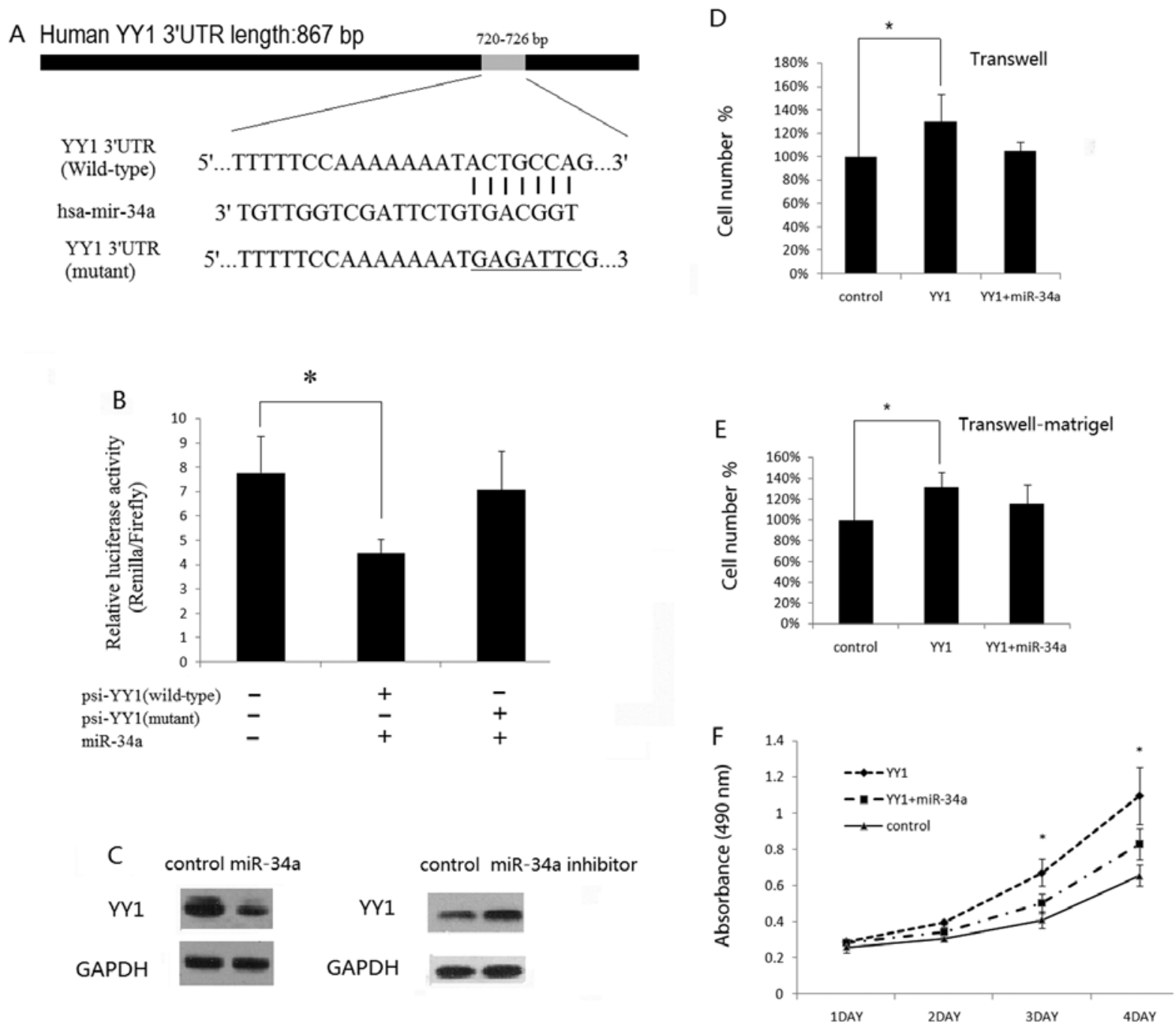

Figure 2. YY1 is a direct target of miR-34a, and overexpression of miR-34a decreases the YY1-induced invasive or proliferative capacity of RCC cells in vitro. (A) Sequence alignments of miR-34a with wild-type and mutant forms of YY1 3'UTR. (B) ACHN cells were transfected with firefly luciferase transcript containing either the wild-type or mutant form of YY1 3'UTR, in the presence of miR-34a mimics. Luciferase activity was then assessed at $48 \mathrm{~h}$ post-transfection. (C) ACHN cells were transfected with either miR-34a mimics or inhibitors. YY1 protein was decreased in ACHN cells following miR-34a mimic treatment, but was elevated following miR-34a inhibitor treatment. (D-F) Ectopic expression of YY1 in ACHN cells promoted migration (D, measured by Transwell assays), invasion (E, measured by Matrigel Transwell assays) and cell growth (F, measured by MTT assay). However, when YY1 and miR34a mimics were co-transfected into ACHN cells, the oncogenic function of YY1 was markedly impaired. P $<0.01$ analyzed by the Student's t-test.

or invasion of RCC cells. We transfected YY1 overexpression plasmids alone, or co-transfected YY1 plasmids and miR-34a mimics into ACHN cells. Notably, exogenous YY1 expression in ACHN cells promoted the migratory, invasive or proliferative potential of ACHN cells (Fig. 2D-F). Furthermore, constitutively expressed miR-34a rescued the previously aggressive phenotype in ACHN cells initiated by YY1 (Fig. 2D-F), suggesting that YY1 is indeed a functional target of miR-34a.

YY1 modulates miR-34a expression via C/EBP $\alpha$ in human $R C C$. Since YY1 downregulates $\mathrm{C} / \mathrm{EBP} \alpha$ expression through a promoter-dependent manner in HCC cells (26) and $\mathrm{C} / \mathrm{EBP} \alpha$ leads to upregulation of miR-34a expression during granulocytic differentiation (27), we aimed to ascertain whether or not YY1 regulates miR-34a expression via $\mathrm{C} / \mathrm{EBP} \alpha$ in human RCC. We observed that miR-34a was downregulated in the pools of cancer tissues when compared to that of the normal tissues. Moreover, miR-34a displayed an inverse correlation with YY1, but a positive correlation with $\mathrm{C} / \mathrm{EBP} \alpha$ (Fig. 3A and B), suggesting that a low level of miR-34a may contribute to the upregulation of YY1 or the elevated expression of YY1 in turn inhibits miR-34a via $\mathrm{C} / \mathrm{EBP} \alpha$ suppression. We then revealed that YY1 siRNA was able to induce the expression of $\mathrm{C} / \mathrm{EBP} \alpha$ both at the mRNA and protein levels in ACHN cells (Fig. 3C and D). We also found that either $\mathrm{C} / \mathrm{EBP} \alpha$ overexpression or $\mathrm{YY} 1$ knockdown alone in $\mathrm{ACHN}$ cells increased miR-34a expression. However, when $\mathrm{C} / \mathrm{EBP} \alpha$ siRNA was transfected into ACHN cells, the upregulation of miR-34a by si-YY1 was abrogated (Fig. 3E). These data indicate that YY1, $\mathrm{C} / \mathrm{EBP} \alpha$ and miR-34a collaborate to form feedforward loops, which contributes to RCC progression (Fig. 4).

\section{Discussion}

In the cancer-associated miRNAs identified to date, miR-34a has emerged as a robust tumor suppressor with diverse targets 

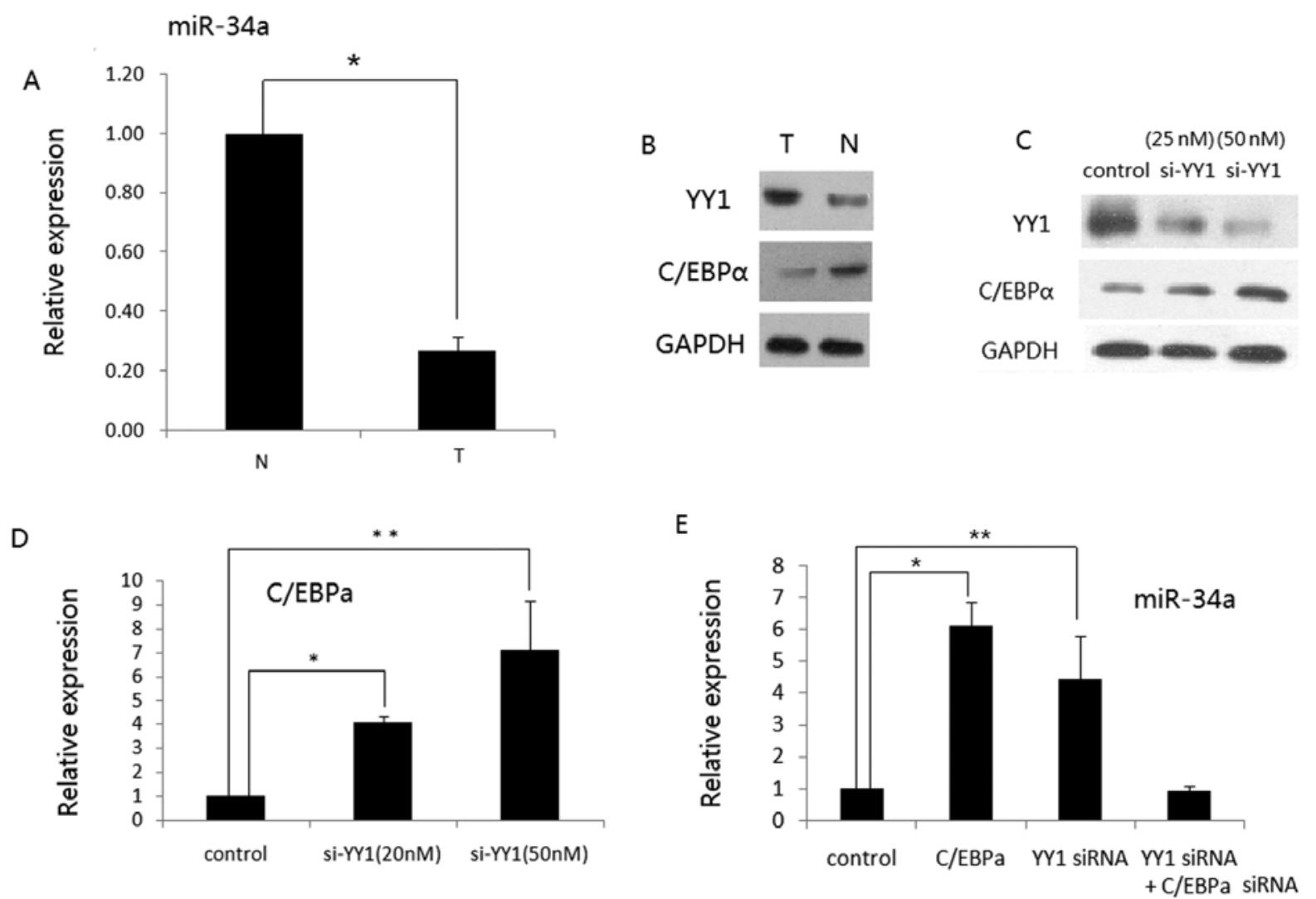

Figure 3. YY1 modulates miR-34a expression via C/EBP $\alpha$ in human RCC. (A) The expression of miR-34a was downregulated in the pool of cancer tissues (T) when compared to that of normal tissues $(\mathrm{N})$. P $<0.01$ analyzed by the Student's t-test. (B) The protein level of YY1 was upregulated while C/EBP $\alpha$ was downregulated in the pools of tumor tissues (T) when compared to that of normal tissues (N). (C and D) YY1 siRNA was able to induce the expression of C/ $\mathrm{EBP} \alpha$ at both the (C) protein and (D) mRNA levels in ACHN cells. (E) Either C/EBP $\alpha$ overexpression or YY1 knockdown alone in ACHN cells increased miR-34a expression. However, when C/EBP $\alpha$ siRNA was transfected into ACHN cells, the upregulation of miR-34a by si-YY1 was abrogated.

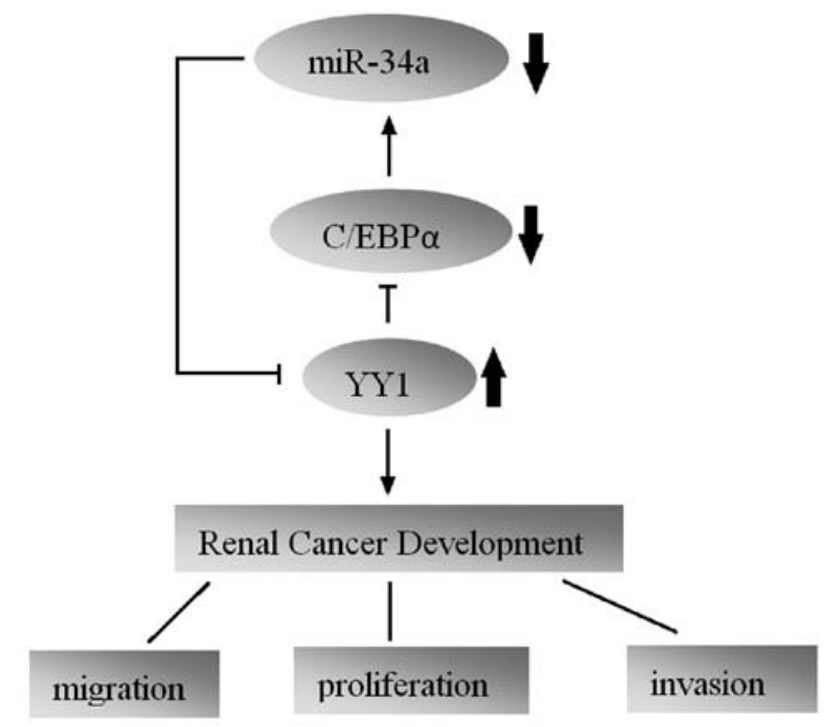

Figure 4. Schematic representation of a positive feedback-loop pathway resulting in YY1 upregulation. Overexpression of YY1 causes repression of $\mathrm{C} / \mathrm{EBP} \alpha$, and the inhibition of $\mathrm{C} / \mathrm{EBP} \alpha$ leads to the suppression of miR-34a. Since YY1 is a direct target of miR-34a, the low level of miR-34a increases the expression of YY1, promoting the aggressiveness of RCC cells.

in multiple types of cancers (15-19). However, the effect of miR-34a and its molecular target in RCC are still largely unknown. Herein, we report that YY1 is a novel target of
miR-34a. We also found that miR34a was downregulated in RCC tissues. Furthermore, overexpression of miR-34a impaired the oncogenic effect of YY1 on cell growth, migration or invasion of RCC cells, suggesting that miR-34a may serve as a potential therapeutic target.

YY1 is a $65-\mathrm{kDa}$ multifunctional zinc-finger transcription factor belonging to the human GLI-Kruppel family of nuclear proteins $(28,29)$. It can bind to the specific DNA consensus sequence, 5'-CGCCATNTT-3', which is present in many promoters and regulates transcriptional activity by either activation or repression $(28,29)$. Therefore, YY1 plays a complicated role in tumor development, largely depending on tissue context, interaction of partners and downstream targets. It was shown that YY1 plays an oncogenic role by activating oncogenes ERBB2 and VEGF, or inhibiting tumor-suppressor genes p53 and E-cadherin $(11,30,31)$. It was also shown that YY1 is able to suppress tumorigenesis via upregulating tumor suppressor genes such as HLJ1 and BRCA1 or by inhibiting c-myc function by direct interaction (32-34).

In this study, we found that YY1 inhibits C/EBP $\alpha$ expression in RCC cells. YY1 siRNA was able to induce the expression of $\mathrm{C} / \mathrm{EBP} \alpha$ both at the mRNA and protein levels in $\mathrm{ACHN}$ cells. Zhang et al found that YY1 suppresses the expression of $\mathrm{C} / \mathrm{EBP} \alpha$ in a promoter-dependent manner in hepatocellular carcinoma cells (26). It was reported that YY1 functions as a critical component of epigenetic regulatory networks. It could promote the methylation of its target genes by physically 
interacting with SUZ12 and recruiting DNA methyltransferases to the promoter of target genes (35). However, Zhang et al did not find that the downregulation of $\mathrm{C} / \mathrm{EBP} \alpha$ in HCC cells was due to the methylation of the promoter despite the upregulation of C/EBP $\alpha$ expression in HCC cells after Aza treatment. Instead, the interaction of YY1 with the C/EBP $\alpha$ promoter was suppressed after Aza treatment. Of note, Girard et al demonstrated that RAR $\alpha$-PLZF (a fusion protein in acute promyelocytic leukemia) recruits HDAC1 and causes histone $\mathrm{H} 3$ deacetylation at $\mathrm{C} / \mathrm{EBP} \alpha$ target loci, thereby decreasing the expression of C/EBP $\alpha$ target genes (36). Therefore, we hypothesized that the inhibitory effect of YY1 on C/EBP $\alpha$ may involve a complex epigenetic network. However, such a hypothesis needs further investigation.

$\mathrm{C} / \mathrm{EBP} \alpha$ is a basic leucine zipper transcription factor that is expressed in many tissues (37). C/EBP $\alpha$ plays an important role in normal tissue development, namely, in the regulation of cell proliferation and cell differentiation $(38,39)$. Several studies here demonstrated that diverse molecular mechanisms are responsible for C/EBP $\alpha$ inactivation of expression or function in various types of cancers, including liver cancer (26), leukemia (AML and chronic myelogenous leukemia) $(40,41)$ and lung cancer (42). It was also shown that C/EBP $\alpha$ directly regulates miR-34a expression during granulocytic differentiation (27). Noteworthy, we found that these two tumor suppressors C/EBP $\alpha$ and miR-34a were both downregulated in renal tumor tissues. Ectopic expression of C/EBP $\alpha$ in ACHN cells promoted miR-34a transcription. Furthermore, YY1 knockdown led to the upregulation of miR-34a through restoring C/EBP $\alpha$ expression in ACHN cells.

In conclusion, our study first showed a regulatory circuitry in RCC cells that incorporates protein-coding and miRNA genes. We found that YY1 is upregulated in human RCC tumors with an oncogenic function. miR-34a exerts a tumorsuppressive function in RCC through directly suppressing oncogenic YY1. Furthermore, repression of C/EBP $\alpha$ caused by YY1 leads to a further reduction in miR-34a expression, which forms a YY1-C/EBP $\alpha-m i R-34 a$ positive feedback loop. Our study highlights the importance of a novel regulatory circuitry in maintaining the proliferative and aggressive phenotypes of RCC, which may serve as a potential therapeutic target for RCC treatment.

\section{Acknowledgements}

This study was supported by the National Natural Science Foundation of China (nos. 81201884 and 81171883).

\section{References}

1. Liou LS, Shi T, Duan ZH, et al: Microarray gene expression profiling and analysis in renal cell carcinoma. BMC Urol 4: 9, 2004.

2. Siegel R, Naishadham D and Jemal A: Cancer statistics, 2012. CA Cancer J Clin 62: 10-29, 2012.

3. Chow WH, Devesa SS, Warren JL and Fraumeni JF Jr: Rising incidence of renal cell cancer in the United States. JAMA 281 1628-1631, 1999.

4. Amato RJ: Chemotherapy for renal cell carcinoma. Semin Oncol 27: 177-186, 2000.

5. Bukowski RM: Natural history and therapy of metastatic renal cell carcinoma: the role of interleukin-2. Cancer 80: 1198-1220, 1997.
6. Janzen NK, Kim HL, Figlin RA and Belldegrun AS: Surveillance after radical or partial nephrectomy for localized renal cell carcinoma and management of recurrent disease. Urol Clin North Am 30: 843-852, 2003.

7. Castellano G, Torrisi E, Ligresti G, et al: The involvement of the transcription factor Yin Yang 1 in cancer development and progression. Cell Cycle 8: 1367-1372, 2009.

8. Zaravinos A and Spandidos DA: Yin yang 1 expression in human tumors. Cell Cycle 9: 512-522, 2010.

9. Riggs KJ, Saleque S, Wong KK, et al: Yin-yang 1 activates the c-myc promoter. Mol Cell Biol 13: 7487-7495, 1993.

10. Lee JS, Galvin KM, See RH, et al: Relief of YY1 transcriptional repression by adenovirus E1A is mediated by E1A-associated protein p300. Genes Dev 9: 1188-1198, 1995.

11. Gronroos E, Terentiev AA, Punga T and Ericsson J: YY1 inhibits the activation of the p53 tumor suppressor in response to genotoxic stress. Proc Natl Acad Sci USA 101: 12165-12170, 2004.

12. Wu S, Murai S, Kataoka K and Miyagishi M: Cooperative regulation of p73 promoter by Yin Yang 1 and E2F1. Nucleic Acids Symp Ser 51: 347-348, 2007.

13. Brodersen $P$ and Voinnet O: Revisiting the principles of microRNA target recognition and mode of action. Nat Rev Mol Cell Biol 10: 141-148, 2009.

14. Kim VN: MicroRNA biogenesis: coordinated cropping and dicing. Nat Rev Mol Cell Biol 6: 376-385, 2005.

15. Fujita Y, Kojima K, Hamada N, et al: Effects of miR-34a on cell growth and chemoresistance in prostate cancer PC3 cells. Biochem Biophys Res Commun 377: 114-119, 2008.

16. Li Y, Guessous F, Zhang Y, et al: MicroRNA-34a inhibits glioblastoma growth by targeting multiple oncogenes. Cancer Res 69: 7569-7576, 2009.

17. Wei JS, Song YK, Durinck S, et al: The MYCN oncogene is a direct target of miR-34a. Oncogene 27: 5204-5213, 2008.

18. Welch C, Chen Y and Stallings RL: MicroRNA-34a functions as a potential tumor suppressor by inducing apoptosis in neuroblastoma cells. Oncogene 26: 5017-5022, 2007.

19. Li N, Fu H, Tie Y, et al: miR-34a inhibits migration and invasion by down-regulation of c-Met expression in human hepatocellular carcinoma cells. Cancer Lett 275: 44-53, 2009.

20. Tazawa H, Tsuchiya N, Izumiya M and Nakagama H: Tumorsuppressive miR-34a induces senescence-like growth arrest through modulation of the E2F pathway in human colon cancer cells. Proc Natl Acad Sci USA 104: 15472-15477, 2007.

21. Chang TC, Wentzel EA, Kent OA, et al: Transactivation of miR-34a by 53 broadly influences gene expression and promotes apoptosis. Mol Cell 26: 745-752, 2007.

22. Liu C, Kelnar K, Liu B, et al: The microRNA miR-34a inhibits prostate cancer stem cells and metastasis by directly repressing CD44. Nat Med 17: 211-215, 2011.

23. Akao Y, Noguchi S, Iio A, Kojima K, Takagi T and Naoe T: Dysregulation of microRNA-34a expression causes drugresistance to 5-FU in human colon cancer DLD-1 cells. Cancer Lett 300: 197-204, 2011.

24. Huang GL, Zhang XH, Guo GL, et al: Clinical significance of miR-21 expression in breast cancer: SYBR-Green I-based real-time RT-PCR study of invasive ductal carcinoma. Oncol Rep 21: 673-679, 2009.

25. Livak KJ and Schmittgen TD: Analysis of relative gene expression data using real-time quantitative PCR and the 2(-Delta Delta C(T)) method. Methods 25: 402-408, 2001.

26. Zhang S, Jiang T, Feng L, et al: Yin Yang-1 suppresses differentiation of hepatocellular carcinoma cells through the downregulation of CCAAT/enhancer-binding protein alpha. J Mol Med 90: 1069-1077, 2012.

27. Pulikkan JA, Peramangalam PS, Dengler V, et al: C/EBPalpha regulated microRNA-34a targets E2F3 during granulopoiesis and is down-regulated in AML with CEBPA mutations. Blood 116: 5638-5649, 2010.

28. Shi Y, Seto E, Chang LS and Shenk T: Transcriptional repression by YY1, a human GLI-Kruppel-related protein, and relief of repression by adenovirus E1A protein. Cell 67: 377-388, 1991.

29. Galvin KM and Shi Y: Multiple mechanisms of transcriptional repression by YY1. Mol Cell Biol 17: 3723-3732, 1997.

30. Tong ZT, Cai MY, Wang XG, et al: EZH2 supports nasopharyngeal carcinoma cell aggressiveness by forming a co-repressor complex with HDAC1/HDAC2 and Snail to inhibit E-cadherin. Oncogene 31: 583-594, 2012.

31. de Nigris F, Crudele V, Giovane A, et al: CXCR4/YY1 inhibition impairs VEGF network and angiogenesis during malignancy. Proc Natl Acad Sci USA 107: 14484-14489, 2010. 
32. Lee MH, Lahusen T, Wang RH, et al: Yin Yang 1 positively regulates BRCA1 and inhibits mammary cancer formation. Oncogene 31: 116-127, 2012.

33. Austen M, Cerni C, Luscher-Firzlaff JM and Luscher B: YY1 can inhibit c-Myc function through a mechanism requiring DNA binding of YY1 but neither its transactivation domain nor direct interaction with c-Myc. Oncogene 17: 511-520, 1998.

34. Wang CC, Tsai MF, Dai TH, et al: Synergistic activation of the tumor suppressor, HLJ1, by the transcription factors YY1 and activator protein 1. Cancer Res 67: 4816-4826, 2007.

35. Samarakoon R and Higgins PJ: Integration of non-SMAD and SMAD signaling in TGF-betal-induced plasminogen activator inhibitor type-1 gene expression in vascular smooth muscle cells. Thromb Haemost 100: 976-983, 2008.

36. Girard N, Tremblay M, Humbert M, et al: RARalpha-PLZF oncogene inhibits C/EBPalpha function in myeloid cells. Proc Natl Acad Sci USA 110: 13522-13527, 2013.

37. Schuster MB and Porse BT: C/EBPalpha: a tumour suppressor in multiple tissues? Biochim Biophys Acta 1766: 88-103, 2006.
38. Lopez RG, Garcia-Silva S, Moore SJ, et al: C/EBPalpha and beta couple interfollicular keratinocyte proliferation arrest to commitment and terminal differentiation. Nat Cell Biol 11: 1181-1190, 2009.

39. Ramji DP and Foka P: CCAAT/enhancer-binding proteins: structure, function and regulation. Biochem J 365: 561-575, 2002.

40. Pabst T and Mueller BU: Complexity of CEBPA dysregulation in human acute myeloid leukemia. Clin Cancer Res 15: 5303-5307, 2009.

41. Perrotti D, Cesi V, Trotta R, et al: BCR-ABL suppresses C/EBPalpha expression through inhibitory action of hnRNP E2. Nat Genet 30: 48-58, 2002.

42. Sato A, Yamada N, Ogawa Y and Ikegami M: CCAAT/enhancerbinding protein-alpha suppresses lung tumor development in mice through the p38alpha MAP kinase pathway. PloS One 8: e57013, 2013. 